\title{
Effects of mitochondrial haplogroup N9a on type 2 diabetes mellitus and its associated complications
}

\author{
QING NIU, WANLIN ZHANG, HAILING WANG, XIAOMIN GUAN, JIANXIN LU and WEI LI
}

\author{
Zhejiang Provincial Key Laboratory of Medical Genetics, Department of Biochemistry and Molecular Biology, \\ Wenzhou Medical University School of Laboratory Medicine and Life Sciences, Wenzhou, Zhejiang 325025, P.R. China
}

Received January 29, 2015; Accepted July 13, 2015

DOI: 10.3892/etm.2015.2751

\begin{abstract}
A case-control study was conducted with the aim of identifying the predominant haplogroups associated with type 2 diabetes mellitus (T2DM) and its complications. In addition, the role of N9a in T2DM risk and complications was analyzed. Sequencing of the entire mitochondrial DNA was conducted in 235 patients and 244 controls in cohort 1 , and six haplogroups (F, B4, D4, D5, M8a and N9a) associated with T2DM were classified. The frequency of N9a was further determined in cohort 2 (440 patients and 244 controls) and examined in two combined cohorts, including 675 patients with T2DM and 649 non-diabetic controls. Multivariate logistic regression analysis and association analysis were performed to investigate the association between genotypes, T2DM and diabetic nephropathy. M8a [P=0.011; odds ratio (OR), 3.49; 95\% confidence interval (CI), 1.26-9.69] and haplogroup N9a $(\mathrm{P}=0.023$; OR, 2.60; 95\% CI, 1.11-6.05) were associated with an increased risk of T2DM. The frequency of N9a was higher in T2DM patients compared with that in the controls $(6.2 \%$ vs. $4.3 \%)$ and associated with a mild risk $(\mathrm{P}=0.10$; $\mathrm{OR}, 1.51$; 95\% CI, 0.92-2.49). N9a was significantly associated with an increased risk of diabetic nephropathy $(\mathrm{P}=0.024$; OR, 2.15; 95\% CI, 1.11-4.19). Previous findings of N9a being protective against T2DM were not replicated in the present study, although this haplogroup was associated with an increased risk of diabetic nephropathy.
\end{abstract}

Correspondence to: Professor Jianxin Lu or Professor Wei Li, Zhejiang Provincial Key Laboratory of Medical Genetics, Department of Biochemistry and Molecular Biology, Wenzhou Medical University School of Laboratory Medicine and Life Sciences, High Education Park, Wenzhou, Zhejiang 325025, P.R. China

E-mail: jxlu313@163.com

E-mail: liweiwzmc@163.com

\section{Abbreviation: T2DM, type 2 diabetes mellitus}

Key words: type 2 diabetes mellitus, mitochondrial genome, M8a, N9a, diabetic nephropathy

\section{Introduction}

Type 2 diabetes mellitus (T2DM) is a highly prevalent disorder worldwide. Research from the International Diabetes Federation has indicated that the number of people with diabetes will increase from 366 million in 2011 to 552 million by 2030 if no urgent action is taken (1). The risk factors for T2DM include life style and social factors, such as unhealthy diet, physical inactivity, obesity and increasing age. The International Diabetes Federation strategy for the prevention of type 2 diabetes is based on controlling modifiable risk factors and identifies two target groups, each requiring a distinct approach: Individuals at a high risk of developing type 2 diabetes and the entire general population. Plans for the widespread prevention of diabetes should take into account both groups simultaneously. Attention must be paid to the creation of conditions that are conducive to achieving and maintaining an active lifestyle and healthy eating habits. T2DM develops as a consequence of the interaction of environmental factors, such as obesity, unhealthy diet and physical inactivity, with genetic variants, although few such variants have been identified. Genome-wide association studies have been performed to determine the genes associated with the susceptibility to T2DM (2-4), which may lead to novel strategies in support of primary prevention $(1,5)$. Dozens of novel diabetes-associated nuclear loci have been revealed in recent studies (5).

Although the mitochondrial genome is far smaller than the nuclear genome, mitochondrial involvement in the pathogenesis of major common metabolic disorders, including T2DM, has been extensively documented through the observation of dysfunctions in the mitochondrial energy production machinery, including oxidative phosphorylation, from a large number of patients $(6,7)$. The common variants or mitochondrial haplogroups of single mitochondrial DNA (mtDNA) mutations, such as major rearrangements (8) and point mutations (9), may modulate the mitochondrial function and thereby be associated with the development of certain conditions. A T- to -C transition at nucleotide pair 16,189, causing a homopolymeric tract of cytosines at nucleotide pairs $16,184-16,193$, was confirmed to accompany the susceptibility to T2DM in certain ethnic groups $(10,11)$. Furthermore, mitochondrial haplogroups have been suggested to contribute to the susceptibility to T2DM and obesity. For instance, the haplogroups $\mathrm{J} / \mathrm{T}$ and $\mathrm{T}$ were found to be associated with an 
increased risk of diabetes in Europeans $(12,13)$. The M8a (14), B4 and D4 haplogroups (15) have significant connection with T2DM in East Asians. However, the haplogroup N9a has been suggested to confer resistance against T2DM in the Japanese and Korean populations (16) and to be a protective factor against metabolic syndrome in Japanese women (17).

In spite of vigorous efforts to identify genetic factors for the susceptibility to T2DM using genetic association analyses, the majority of studies either failed to report a definitive association between T2DM and variation in the mitochondrial genome or presented conflicting results (18). Owing to the heterogeneity in environmental factors, as well as genetic backgrounds, the susceptibility to T2DM, other metabolic and complex diseases differs widely in different ethnic populations (19). The aforementioned findings, particularly the conflicting observations, which were likely attributable to different populations and environments, or even different genotyping methods (20), may not be applicable to all Han Chinese individuals. It has also been reported that mtDNA backgrounds (haplogroups) modulated diabetes complications rather than modulating T2DM (21). The aim of the present study was to identify mtDNA anomalies associated with T2DM in the Han Chinese population. A two-stage case-control investigation was conducted: Initially, six haplogroups associated with T2DM in East Asians were assessed by sequencing the entire mitochondrial genome. Based on these findings, haplogroup N9a was then focused on and its association with T2DM risk and complications was evaluated.

\section{Materials and methods}

Ethics statement. Written informed consent was obtained from all the participants prior to the study. Clinical evaluations were approved by the Ethics Committee of the First Affiliated Hospital of Wenzhou Medical University (Wenzhou, China) Institutional Review Board (Supplementary 1).

Subjects. For cohorts 1 and 2, unrelated patients of Han Chinese origin were recruited from the First Affiliated Hospital of Wenzhou Medical College. Cohort 1 patients underwent entire mtDNA genome sequencing, then cohort 2 was collected for N9a determination using polymerase chain reaction (PCR) and DNA sequencing. Age- and gender-matched non-diabetic controls were recruited from the Health Screening Center in the same hospital between March 2009 and October 2010. The diagnosis of T2DM was in accordance with the World Health Organization (WHO) 1999 criteria (22) and the China Guideline for Type 2 Diabetes (issued by the Chinese Diabetes Society in 2010) (23). The non-diabetic controls were defined as individuals with a fasting plasma glucose (FPG) level of $<6.1 \mathrm{mmol} / \mathrm{l}$ and no history of diabetes.

Mitochondrial genome analysis in cohort 1. For all the subjects in cohort 1, genomic DNA was extracted from peripheral whole blood using a Blood Genome DNA Extraction Kit (Takara Biotechnology Co. Ltd., Dalian, China). The entire mtDNA of the proband was amplified in 24 overlapping fragments using PCR with sets of light-strand and heavy-strand oligonucleotide primers, as described elsewhere (24). The $25-\mu 1$ reaction mixture contained $50 \mathrm{ng}$ genomic DNA, 5 pmol of each primer, $0.2 \mathrm{mmol} / 1$ of each dNTP, $1.5 \mathrm{mmol} / 1 \mathrm{MgCl}_{2}$, and $1 \mathrm{U}$ Taq DNA Polymerase in a 10x PCR buffer. The cycling conditions were as follows: $95^{\circ} \mathrm{C}$ for $10 \mathrm{~min}$, followed by 32 cycles of denaturation at $94^{\circ} \mathrm{C}$ for $20 \mathrm{sec}$, annealing at $60^{\circ} \mathrm{C}$ for $30 \mathrm{sec}$, and extension at $72^{\circ} \mathrm{C}$ for $30 \mathrm{sec}$, with a final extension at $72^{\circ} \mathrm{C}$ for $10 \mathrm{~min}$. The fragments were purified and subsequently analyzed by direct sequencing with an ABI 3730xl (Applied Biosystems Life Technologies, Beijing, China) automated DNA sequencer at the Beijing Genomics Institute (Beijing, China). The resultant sequence data were compared with the updated consensus Cambridge reference sequence (GenBank no., NC_012920). A total of 20 polymorphic sites were selected to define six mitochondrial haplogroups [M8a (20), B4, D4 (12), F, D5, N9a (13)] that had been previously reported to be associated with T2DM in the East Asian population.

Haplogroup N9a determination in cohort 2. In the second stage of the study, haplogroup N9a was evaluated for all participants in cohort 2 by means of three polymorphic sites (5231A, 12358G and 12372A) that had been widely accepted for the nomenclature in previous studies $(16,25)$. Two fragments spanning nt 4,485-6,642 and nt 10,672-12,772 of mtDNA were amplified for the detection of the polymorphic sites using PCR with primers Set1 and Set2, respectively. The primer sequences were as follows: Set1 forward, 5'-ACTAAT TAATCCCCTGGCCC-3', and reverse, 5'-ATTCCGAAGCCT GGTAGGAT-3'; Set2 forward, 5'-GCCATACTAGTCTTT GCCGC-3', and reverse, 5'-AGAAGGTTATAAAGAAGA AGGTTATAATTCCTACG-3'.

The fragments were purified and then analyzed by direct sequencing. The sequence analysis was processed using the online tool, Standard Nucleotide BLAST (http://blast. ncbi.nlm.nih.gov/), to identify the nucleotide changes from the counterparts of the Cambridge reference sequence for human mtDNA.

Analysis of clinical characteristics and T2DM complications. According to the results of haplogroup N9a determination, the T2DM patients were divided into N9a and non-N9a groups. The diabetic chronic complications were diagnosed by diabetologists according to the China Guideline for Type 2 Diabetes, as follows: i) Diabetic retinopathy was assessed using fundoscopy through dilated pupils and/or fluorescence angiography; ii) diabetic nephropathy was defined by the value of urinary albumin excretion (microalbuminuria, 30-300 mg/24 h; and macroalbuminuria, $>300 \mathrm{mg} / 24 \mathrm{~h}$ ); iii) diabetic neuropathy was diagnosed based on the presented symptoms and a foot examination (appearance of the feet, ankle reflexes, vibration testing and pressure sensation); and iv) macrovascular disease and ischemic heart disease were diagnosed by clinical history, and/or ischemic electrocardiographic alterations. Peripheral arterial occlusive diseases (PAOD), including atherosclerosis obliterans and cerebrovascular disease, were established on the basis of the patient's medical history, physical examinations and laser Doppler flowmetry (26).

Statistical analysis. Quantitative clinical data, including age, body mass index (BMI), systolic and diastolic blood pressure 
Table I. Summary of clinical characteristics of subjects in cohort 1.

\begin{tabular}{lccc}
\hline Variables & T2DM cases $(\mathrm{n}=235)$ & Controls $(\mathrm{n}=244)$ & P-value \\
\hline Age (years) & $62.2 \pm 11.6$ & $60.9 \pm 10.7$ & $>0.050$ \\
Gender, F/M $(\%)$ & $54.2 / 45.8$ & $57.4 / 42.6$ & $>0.050$ \\
BMI $\left(\mathrm{kg} / \mathrm{m}^{2}\right)$ & $24.0 \pm 3.5$ & $23.6 \pm 3.0$ & $>0.050$ \\
SBP $(\mathrm{mmHg})$ & $145 \pm 24$ & $127 \pm 18$ & $<0.001$ \\
DBP $(\mathrm{mmHg})$ & $80 \pm 12$ & $80 \pm 11$ & $>0.050$ \\
TC $(\mathrm{mmol} / \mathrm{l})$ & $4.42 \pm 1.08$ & $5.27 \pm 1.06$ & $<0.001^{\mathrm{a}}$ \\
TG $(\mathrm{mmol} / \mathrm{l})$ & $1.78 \pm 1.78$ & $1.47 \pm 1.35$ & $<0.050^{\mathrm{a}}$ \\
HDL $(\mathrm{mmol} / \mathrm{l})$ & $1.16 \pm 0.44$ & $1.33 \pm 0.33$ & $<0.001^{\mathrm{a}}$ \\
FPG $(\mathrm{mmol} / \mathrm{l})$ & $8.99 \pm 3.84$ & $5.71 \pm 1.14$ & $<0.001^{\mathrm{a}}$ \\
\hline
\end{tabular}

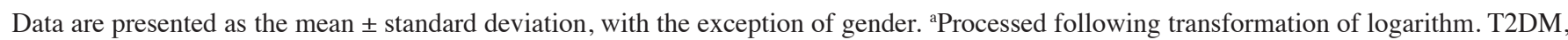
type 2 diabetes mellitus; F/M, female/male; BMI, body mass index; SBP, systolic blood pressure; DBP, diastolic blood pressure; TC, total cholesterol; TG, triglycerides; HDL, high-density lipoprotein; FPG, fasting plasma glucose.

Table II. Haplogroup distribution in patients with T2DM and controls in cohort 1.

\begin{tabular}{lcccr}
\hline & T2DM cases $(\mathrm{n}=235)$, & Controls $(\mathrm{n}=244)$, & & \\
Haplogroup $^{\mathrm{a}}$ & $\mathrm{n}(\%)$ & $\mathrm{n}(\%)$ & P-value & OR \\
\hline F & $31(13.2)$ & $27(11.1)$ & 0.476 & 1.22 \\
B4 & $21(8.9)$ & $15(6.1)$ & 0.247 & 1.50 \\
D4 & $32(13.6)$ & $33(13.5)$ & 0.976 & $0.70-2.12$ \\
D5 & $17(7.2)$ & $19(7.8)$ & 0.819 & 0.92 \\
M8a & $16(6.8)$ & $5(2.0)$ & 0.011 & 3.49 \\
N9a & $19(8.1)$ & $8(3.3)$ & 0.023 & $0.60-1.70$ \\
\hline
\end{tabular}

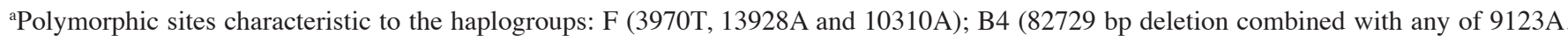
4280A, 15346A, 13942G and 4048A); D4 (4883T, 5178A and 3010A); D5 (4883T, 5178A and 10397G); M8a (15487T and 8684T); N9a (5231 A, 12358G and 12372A). T2DM, type 2 diabetes mellitus; OR, odds ratio; 95\% CI, 95\% confidence interval.

(SBP and DBP, respectively), total cholesterol (TC), triglycerides (TG), high-density lipoprotein (HDL) and FPG were represented as the mean \pm standard deviation. These variables were compared between patients with T2DM and non-diabetic individuals by one-way analysis of variance. Non-normal distribution data were processed following transformation of the logarithm into normal distribution. Gender was described as female or male and compared using a $\chi^{2}$ test. Haplogroups and complications were represented as numbers of patients and percentages. The frequencies of haplogroups were compared using a $\chi^{2}$ test. For the two cohorts together, multivariate logistic-regression analysis with adjustment for age, gender and BMI was used in the analysis of N9a presence, as well as in the comparison of complications. The P-value, odds ratio (OR) and $95 \%$ confidence interval $(\mathrm{CI})$ were calculated. $\mathrm{P}<0.05$ was considered to indicate a statistically significant difference. Statistical analysis was processed using SPSS software (version 17.0; SPSS Inc., Chicago, IL, USA).

\section{Results}

Mitochondrial genome analysis in cohort 1. During the initial stage of the study (i.e. the mitochondrial haplogroup analysis in cohort 1), 235 patients with T2DM and 244 non-diabetic controls were recruited and matched by age, gender and BMI. The clinical characteristics of all the subjects are described in Table I. The data demonstrated that the levels of SBP, TG and FPG were significantly higher in patients with T2DM compared with those in controls $(\mathrm{P}<0.05)$, whereas the level of HDL was lower in the patients with T2DM compared with that in the controls $(\mathrm{P}<0.05)$, indicating the typical clinical manifestation of T2DM and metabolic syndrome.

Following the clinical evaluation and genomic DNA preparation, entire-mitochondrial-genome sequencing was performed for the classification of six mitochondrial haplogroups (F, B4, D4, D5, M8a and N9a) associated with T2DM or metabolic syndrome in East Asians. Consistent with a previous report (14), it was identified that M8a was replicated in the present study with a higher frequency in the T2DM population than in controls $(\mathrm{P}=0.011)$. In addition, M8a was found to be associated with a significantly increased risk of developing T2DM (OR, 3.49; 95\% CI, 1.26-9.69), while the F, B4, D4 and D5 haplogroups did not correlate with T2DM in the Han Chinese population (Table II). Notably, haplogroup N9a, which had been reported to be associated with resistance to T2DM in a Japanese population $(16,17)$, 
Table III. Summary of clinical characteristics of the subjects in cohort 2.

\begin{tabular}{lccc}
\hline Variables & T2DM cases $(\mathrm{n}=440)$ & Controls $(\mathrm{n}=405)$ & P-value \\
\hline Age (years) & $60.0 \pm 13.3$ & $57.1 \pm 11.7$ & $<0.001$ \\
Gender F/M (\%) & $199 / 241$ & $221 / 184$ & $<0.010$ \\
BMI $\left(\mathrm{kg} / \mathrm{m}^{2}\right)$ & $24.3 \pm 3.29$ & $22.4 \pm 2.35$ & $<0.001$ \\
SBP $(\mathrm{mmHg})$ & $142 \pm 24$ & $117 \pm 12$ & $<0.001$ \\
DBP $(\mathrm{mmHg})$ & $80 \pm 12$ & $74 \pm 8$ & $<0.001$ \\
TC $(\mathrm{mmol} / \mathrm{l})$ & $4.64 \pm 1.26$ & $4.60 \pm 0.89$ & $>0.050^{\mathrm{a}}$ \\
TG $(\mathrm{mmol} / \mathrm{l})$ & $1.72 \pm 1.74$ & $1.09 \pm 0.51$ & $<0.001^{\mathrm{a}}$ \\
HDL $(\mathrm{mmol} / \mathrm{l})$ & $1.21 \pm 0.42$ & $1.42 \pm 0.30$ & $<0.001^{\mathrm{a}}$ \\
FPG $(\mathrm{mmol} / \mathrm{l})$ & $9.27 \pm 5.24$ & $4.81 \pm 0.68$ & $20(4.9)$
\end{tabular}

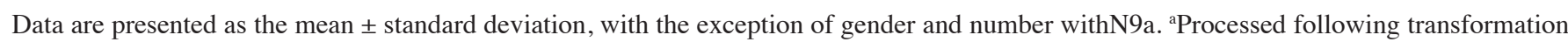
of logarithm. T2DM, type 2 diabetes mellitus; F/M, female/male; BMI, body mass index; SBP, systolic blood pressure; DBP, diastolic blood pressure; TC, total cholesterol; TG, triglycerides; HDL, high density lipoprotein; FPG, fasting plasma glucose.

Table IV. Multivariate logistic regression analysis in the combination of cohorts 1 and 2.

\begin{tabular}{lcccc}
\hline T2DM cases $(\mathrm{n}=675)$, & Controls $(\mathrm{n}=649)$, \\
$\mathrm{n}(\%)$ & $\mathrm{n}(\%)$ & P-value & OR & $95 \%$ CI \\
\hline N9a & $42(6.2)$ & $28(4.3)$ & 0.123 & $\begin{array}{l}1.47 \\
1.51^{\mathrm{a}}\end{array}$ \\
& & & $0.102^{\mathrm{a}}$ & $0.90-2.40$ \\
& & & $0.92-2.49^{\mathrm{a}}$ \\
\hline
\end{tabular}

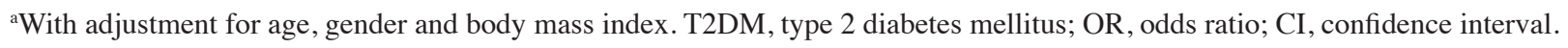

was associated with an increased risk of T2DM in the present population ( $\mathrm{P}=0.023$; OR, 2.60; $95 \% \mathrm{CI}, 1.11-6.05)$.

Haplogroup N9a determination in cohort 2. In order to verify the aforementioned contradictory result for haplogroup N9a, a further 440 patients with T2DM and 405 non-diabetic controls were enlisted as cohort 2 for the second stage of the current study. Their clinical characteristics are listed in Table III. The fragments harboring the mtDNA single nucleotide polymorphisms that define haplogroup N9a (5231A, 12358G and 12372A) $(16,24)$ were amplified using PCR and subsequently analyzed by direct sequencing. The identification of N9a was conducted based on the BLAST results. The N9a haplogroup was again more frequent in the T2DM group than in the non-diabetic controls, although the difference was not statistically significant (5.2 vs. $4.9 \%$; P>0.05). Furthermore, no significant difference was observed in the N9a frequency in the control group between cohort 1 and cohort $2(\mathrm{P}=0.31$; data not shown).

Clinical characteristics and T2DM complications. In order to further characterize the role of the N9a haplogroup, the association between N9a and the risk of T2DM was comprehensively examined by combining the results of cohort 1 and cohort 2 using multivariate logistic regression analysis with adjustment for gender, age and BMI (Table IV). The frequency of N9a in patients with T2DM was found to be $6.2 \%$, which demonstrated a marginal increase compared with the $4.3 \%$ frequency in the controls; however, the difference was not statistically significant
( $\mathrm{P}=0.10$; OR, 1.51; 95\% CI, 0.92-2.49). Thus, based on the data of the present study, it remains uncertain whether N9a is correlated with an increased risk of T2DM; however, N9a clearly was not protective against T2DM in the Han Chinese population.

The association of the N9a haplogroup with the risk of specific complications in patients with T2DM was also investigated. The clinical characteristics of N9a and non-N9a patients are summarized in Table V. No statistically significant differences were identified between the two groups in gender ratio, age, BMI, blood pressure, TC, TG, HDL and FPG ( $\mathrm{P}>0.05)$. Subsequently, a multivariate logistic regression model was established to examine the association between the N9a haplogroup and the risk of T2DM complications. The risk of diabetic nephropathy in N9a patients significantly increased $(\mathrm{P}=0.006$; OR, 2.35; 95\% CI, 1.25-4.44) even with adjustment for age, gender and $\mathrm{BMI}(\mathrm{P}=0.024$; OR, 2.15; 95\% CI, 1.11-4.19). Diabetic neuropathy, diabetic foot and macrovascular disease also tended to have a higher incidence in patients with N9a compared with those without N9a, although the differences were not statistically significant (Table VI). This result suggested a new hypothesis that N9a may confer a genetic predisposition to the development of T2DM complications, particularly diabetic nephropathy.

\section{Discussion}

In the present study, a case-control investigation was conducted to explore the effects of mtDNA haplogroups on T2DM risk 
Table V. Clinical characteristics for patients with type 2 diabetes mellitus with and without the N9a haplogroup.

\begin{tabular}{lccc}
\hline Variables & N9a & Non-N9a \\
$\mathrm{n}=42$ & 633 & $334 / 299$ & 0.962 \\
\hline Gender (F/M) & $22 / 20$ & $61.3 \pm 13.0$ & 0.770 \\
Age (years) & $61.9 \pm 10.3$ & $24.2 \pm 3.4$ & 0.603 \\
BMI $\left(\mathrm{kg} / \mathrm{m}^{2}\right)$ & $24.5 \pm 3.5$ & $142.7 \pm 24.4$ & 0.550 \\
SBP $(\mathrm{mmHg})$ & $145.1 \pm 24.5$ & $80.1 \pm 11.7$ & 0.910 \\
DBP $(\mathrm{mmHg})$ & $80.3 \pm 11.5$ & $8.1 \pm 4.3$ & $0.311^{\mathrm{a}}$ \\
FPG $(\mathrm{mmol} / \mathrm{l})$ & $8.5 \pm 4.9$ & $4.4 \pm 1.4$ & $0.506^{\mathrm{a}}$ \\
TC $(\mathrm{mmol} / \mathrm{l})$ & $4.3 \pm 1.2$ & $1.3 \pm 1.0$ & $0.101^{\mathrm{a}}$ \\
TG $(\mathrm{mmol} / \mathrm{l})$ & $1.1 \pm 1.1$ & $1.1 \pm 0.4$ & $0.992^{\mathrm{a}}$ \\
HDL $(\mathrm{mmol} / \mathrm{l})$ & $1.1 \pm 0.5$ & & \\
\hline
\end{tabular}

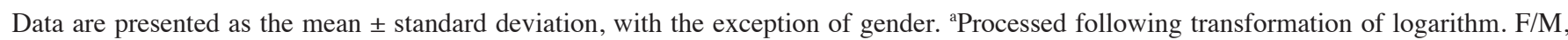
female/male; BMI, body mass index; SBP, systolic blood pressure; DBP, diastolic blood pressure; TC, total cholesterol; TG, triglycerides; HDL, high density lipoprotein; FPG, fasting plasma glucose.

Table VI. Association of haplogroup N9a with the risk of diabetic complications.

\begin{tabular}{|c|c|c|c|c|c|}
\hline Complication & $\begin{array}{c}\text { N9a }(\mathrm{n}=42) \\
\mathrm{N}(\%)\end{array}$ & $\begin{array}{c}\text { Non-N9a }(\mathrm{n}=633) \\
\text { N }(\%)\end{array}$ & P-value & OR & $95 \% \mathrm{CI}$ \\
\hline Diabetic retinopathy & $20(47.6)$ & $307(48.5)$ & $\begin{array}{l}0.904 \\
0.915^{\mathrm{a}}\end{array}$ & $\begin{array}{l}0.96 \\
0.97\end{array}$ & $\begin{array}{l}0.52-1.80 \\
0.51-1.83\end{array}$ \\
\hline Diabetic nephropathy & $19(45.2)$ & $164(25.9)$ & $\begin{array}{l}0.006 \\
0.024^{\mathrm{a}}\end{array}$ & $\begin{array}{l}2.35 \\
2.15\end{array}$ & $\begin{array}{l}1.25-4.44 \\
1.11-4.19\end{array}$ \\
\hline Diabetic neuropathy & $26(61.9)$ & $312(49.3)$ & $\begin{array}{l}0.116 \\
0.207^{\mathrm{a}}\end{array}$ & $\begin{array}{l}1.67 \\
1.52\end{array}$ & $\begin{array}{l}0.88-3.17 \\
0.79-2.92\end{array}$ \\
\hline Diabetic foot & $10(23.8)$ & $86(13.6)$ & $\begin{array}{l}0.066 \\
0.061^{\mathrm{a}}\end{array}$ & $\begin{array}{l}1.99 \\
2.09\end{array}$ & $\begin{array}{l}0.94-4.19 \\
0.97-4.54\end{array}$ \\
\hline Macrovascular disease & $23(54.8)$ & $301(47.6)$ & $\begin{array}{l}0.365 \\
0.484^{\mathrm{a}}\end{array}$ & $\begin{array}{l}1.34 \\
1.27\end{array}$ & $\begin{array}{l}0.71-2.50 \\
0.65-2.51\end{array}$ \\
\hline
\end{tabular}

${ }^{a}$ Multivariate logistic regression analysis with adjustment for age, gender and body mass index.

and complications in a Chinese population. The data suggested that mitochondrial haplogroup N9a was not protective against T2DM and may even have been associated with an increased risk of T2DM in the Han Chinese population. Furthermore, the current data demonstrated that N9a was associated with an increased risk of diabetic nephropathy in this population.

Mitochondrial haplogroups defined by common polymorphisms are known to be associated with the risk of a variety of diseases, including Leber's hereditary optic neuropathy (27), Alzheimer's disease (28), cardiovascular disease (29) and T2DM (14-17). It was hypothesized that mitochondrial haplogroups confer the genetic predisposition to develop T2DM when an individual is exposed continuously to environmental factors. In the European population, a trend of increased T2DM risk was seen with haplogroup J $(12,13)$. A study in a Japanese population indicated that M8a and B4c contributed to susceptibility to T2DM or obesity (14), whereas N9a conferred resistance to T2DM in a combined Japanese and Korean population (16), and provided protection against meta- bolic syndrome in Japanese women (17). By contrast, a recent study in a Taiwanese population with Chinese ethnic backgrounds revealed that subjects harboring the haplogroup B4 had significant morbidity from diabetes, whereas subjects harboring D4 had borderline resistance to T2DM development; however, subjects with N9a did not present significant morbidity or resistance (15).

Similar to previous findings, the present study, which was performed in a Han Chinese population, did not identify a protective role of haplogroup N9a against T2DM. Although the current study was conducted in a smaller population than that of the Japanese study (16), the finding that N9a had a tendency to be associated with an increased risk of T2DM is still noteworthy. The results supported the idea that the mitochondrial haplogroup, which is involved in the regulation of mitochondrial function, is modulated by other factors, including environmental factors and nuclear-encoded genes $(30,31)$. Evidence from transmitochondrial cytoplasmic hybrid (cybrid) cell assays (32) revealed no significant difference in 
mitochondrial function in terms of adenosine triphosphate generation, reactive oxygen species production, mitochondrial membrane potential or cellular dehydrogenase activity among cybrid cells harboring each of the three haplogroups N9a, D5 and F in an identical nuclear background; however, a distinctive nuclear gene expression pattern emerged. It was concluded that defective nuclear compensation under certain circumstances may lead to the development of T2DM. Certain nuclear genetic backgrounds may thus modify the role of N9a in the susceptibility to T2DM in the Han Chinese population.

Another concern in the present study was the role of mtDNA variants or haplogroups in modulating susceptibility to developing diabetes complications. It has been reported that different mitochondrial haplogroups are significantly associated with an increased risk of specific diabetes complications in the European population (18), such as haplogroup H with retinopathy, H3 with neuropathy, U3 with nephropathy and $\mathrm{V}$ with renal failure. In the present study, haplogroup N9a was associated with an increased risk of diabetic neuropathy, macrovascular disease and diabetic foot and, in particular, a significantly increased risk of diabetic nephropathy, even following multivariate logistic regression analysis with adjustment for gender, age and BMI. These results indicated that a putative nuclear-encoded gene underlying haplogroup N9a may be involved in the initiation or exacerbation of diabetic nephropathy in the Han Chinese population. In addition, the current study provided further evidence that N9a is not a protective factor for T2DM in this population.

In conclusion, N9a was not protective against T2DM in a Han Chinese population, but was associated with an increased risk of diabetic nephropathy. This result suggested that the effect of N9a is modulated by interactions between mitochondrial haplogroups, nuclear polymorphisms and environmental factors.

\section{Acknowledgements}

The authors would like to thank Dr Wei Ye, Dr Min Li, Ms. Mei Zhang and Dr Weixing Li for their kind assistance in the recruitment of the subjects, and in mitochondrial genome analysis.

\section{References}

1. Whiting DR, Guariguata L, Weil C and Shaw J: IDF diabetes atlas: Global estimates of the prevalence of diabetes for 2011 and 2030. Diabetes Res Clin Pract 94: 311-321, 2011.

2. Scott LJ, Mohlke KL, Bonnycastle LL, Willer CJ,Li Y, Duren WL, Erdos MR, Stringham HM, Chines PS, Jackson AU, et al: A genome-wide association study of type 2 diabetes in Finns detects multiple susceptibility variants. Science 316: 1341-1345, 2007.

3. Yasuda K, Miyake K, Horikawa Y, Hara K, Osawa H, Furuta H, Hirota Y, Mori H, Jonsson A, Sato Y, et al: Variants in KCNQ1 are associated with susceptibility to type 2 diabetes mellitus. Nat Genet 40: 1092-1097, 2008 .

4. Cho YS, Chen CH, Hu C, Long J, Ong RT, Sim X, Takeuchi F, Wu Y, Go MJ, Yamauchi T, et al: Meta-analysis of genome-wide association studies identifies eight new loci for type 2 diabetes in east Asians. Nat Genet 44: 67-72, 2011.

5. Hara K, Shojima N, Hosoe J and Kadowaki T: Genetic architecture of type 2 diabetes. Biochem Biophys Res Commun 452: 213-220, 2014

6. Lowell BB and Shulman GI: Mitochondrial dysfunction and type 2 diabetes. Science 307: 384-387, 2005.
7. Petersen KF, Dufour S, Befroy D, Garcia R and Shulman GI: Impaired mitochondrial activity in the insulin-resistant offspring of patients with type 2 diabetes. N Engl J Med 350: 664-671, 2004.

8. Ballinger SW, Shoffner JM, Hedaya EV, Trounce I, Polak MA, Koontz DA and Wallace DC: Maternally transmitted diabetes and deafness associated with a $10.4 \mathrm{~kb}$ mitochondrial DNA deletion. Nat Genet 1: 11-15, 1992.

9. van den Ouweland JM, Lemkes HH, Ruitenbeek W, Sandkuijl LA, de Vijlder MF, Struyvenberg PA, van de Kamp JJ and Maassen JA: Mutation in mitochondrial tRNA (Leu) (UUR) gene in a large pedigree with maternally transmitted type II diabetes mellitus and deafness. Nat Genet 1: 368-371, 1992.

10. Liou CW, Lin TK, Huei Weng H, Lee CF, Chen TL, Wei YH, Chen SD, Chuang YC, Weng SW and Wang PW: A common mitochondrial DNA variant and increased body mass index as associated factors for development of type 2 diabetes: Additive effects of genetic and environmental factors. J Clin Endocrinol Metab 92: 235-239, 2007.

11. Park KS, Chan JC, Chuang LM, Suzuki S, Araki E, Nanjo K, Ji L, Ng M, Nishi M and Furuta H: A mitochondrial DNA variant at position 16189 is associated with type 2 diabetes mellitus in Asians. Diabetologia 51: 602-608, 2008.

12. Crispim D, Canani LH, Gross JL, Tschiedel B, Souto KE and Roisenberg I: The European-specific mitochondrial cluster J/T could confer an increased risk of insulin-resistance and type 2 diabetes: An analysis of the m.4216T $>\mathrm{C}$ and $\mathrm{m} .4917 \mathrm{~A}>\mathrm{G}$ variants. Ann Hum Genet 70: 488-495, 2006.

13. Mohlke KL, Jackson AU, Scott LJ, Peck EC, Suh YD, Chines PS Watanabe RM, Buchanan TA, Conneely KN, Erdos MR, et al: Mitochondrial polymorphisms and susceptibility to type 2 diabetes-related traits in Finns. Hum Genet 118: 245-254, 2005.

14. Guo LJ, Oshida Y, Fuku N, Takeyasu T,Fujita Y, Kurata M, Sato Y, Ito $\mathrm{M}$ and Tanaka $\mathrm{M}$ : Mitochondrial genome polymorphisms associated with type-2 diabetes or obesity. Mitochondrion 5: 15-33, 2005.

15. Liou CW, Chen JB, Tiao MM, Weng SW, Huang TL, Chuang JH, Chen SD, Chuang YC, Lee WC, Lin TK and Wang PW: Mitochondrial DNA coding and control region variants as genetic risk factors for type 2 diabetes. Diabetes 61: 2642-2651, 2012.

16. Fuku N, Park KS, Yamada Y, Nishigaki Y, Cho YM, Matsuo H, Segawa T, Watanabe S, Kato K, Yokoi K, et al: Mitochondrial haplogroup N9a confers resistance against type 2 diabetes in Asians. Am J Hum Genet 80: 407-415, 2007.

17. Tanaka M, Fuku N, Nishigaki Y, Matsuo H, Segawa T, Watanabe S, Kato K, Yokoi K, Ito M, Nozawa Y and Yamada Y: Women with mitochondrial haplogroup N9a are protected against metabolic syndrome. Diabetes 56: 518-521, 2007.

18. Saxena R, de Bakker PI, Singer K, Mootha V, Burtt N, Hirschhorn JN, Gaudet D, Isomaa B, Daly MJ, Groop L, et al: Comprehensive association testing of common mitochondrial DNA variation in metabolic disease. Am J Hum Genet 79: 54-61, 2006.

19. Shriner D, Vaughan LK, Padilla MA and Tiwari HK: Problems with genome-wide association studies. Science 316: 1840-1842, 2007.

20. Pei YF, Li J, Zhang L, Papasian CJ and Deng HW: Analyses and comparison of accuracy of different genotype imputation methods. PLoS One 3: e3551, 2008.

21. Achilli A, Olivieri A, Pala M, Hooshiar Kashani B, Carossa V, Perego UA, Gandini F, Santoro A, Battaglia V, Grugni V, et al: Mitochondrial DNA backgrounds might modulate diabetes complications rather than T2DM as a whole. PloS One 6: e21029, 2011.

22. World Health Organization: Definition, diagnosis and classification of diabetes mellitus and its complications: Report of a WHO Consultation. Part 1. Diagnosis and classification of diabetes mellitus. Geneva, World Health Organization, 1999

23. Chinese Diabetes Society: China guideline for type 2 diabetes-Diagnosis and classification of diabetes mellitus. Beijing, Chinese Diabetes Society, 2011.

24. Rieder MJ, Taylor SL, Tobe VO and Nickerson DA: Automating the identification of DNA variations using quality-based fluorescence re-sequencing: Analysis of the human mitochondrial genome. Nucleic Acids Res 26: 967-973, 1998.

25. Guo LJ, Oshida Y, Fuku N, Takeyasu T, Fujita Y, Kurata M, Sato Y, Ito $\mathrm{M}$ and Tanaka $\mathrm{M}$ : Mitochondrial genome polymorphisms associated with type-2 diabetes or obesity. Mitochondrion 5: 15-33, 2005. 
26. Ludyga T, Kuczmik WB, Kazibudzki M, Nowakowski P, Orawczyk T, Glanowski M, Kucharzewski M, Ziaja D, Szaniewski K and Ziaja K: Ankle-brachial pressure index estimated by laser Doppler in patients suffering from peripheral arterial obstructive disease. Ann Vasc Surg 21: 452-457, 2007.

27. Kong QP, Bandelt HJ, Sun C, Yao YG, Salas A, Achilli A Wang CY, Zhong L, Zhu CL, Wu SF, et al: Updating the East Asian mtDNA phylogeny: A prerequisite for the identification of pathogenic mutations. Hum Mol Genet 15: 2076-2086, 2006.

28. Zhang AM, Jia X, Bi R, Salas A, Li S, Xiao X, Wang P, Guo X, Kong QP, Zhang Q and Yao YG: Mitochondrial DNA haplogroup background affects LHON, but not suspected LHON, in Chinese patients. PloS One 6: e27750, 2011.

29. Santoro A, Balbi V, Balducci E, Pirazzini C, Rosini F, Tavano F, Achilli A, Siviero P, Minicuci N, Bellavista E, et al: Evidence for sub-haplogroup h5 of mitochondrial DNA as a risk factor for late onset Alzheimer's disease. PloS One 5: e12037, 2010.
30. Palacín M, Alvarez V, Martín M, Díaz M, Corao AI, Alonso B, Díaz-Molina B,Lozano I, Avanzas P, Morís C, et al: Mitochondrial DNA and TFAM gene variation in early-onset myocardial infarction: Evidence for an association to haplogroup $\mathrm{H}$. Mitochondrion 11: 176-181, 2011.

31. Guan MX, Yan Q, Li X, Bykhovskaya Y, Gallo-Teran J, Hajek P, Umeda N, Zhao H, Garrido G, Mengesha E, et al: Mutation in TRMU related to transfer RNA modification modulates the phenotypic expression of the deafness-associated mitochondrial $12 \mathrm{~S}$ ribosomal RNA mutations. Am J Hum Genet 79: 291-302, 2006.

32. Hwang S, Kwak SH, Bhak J, Kang HS, Lee YR, Koo BK, Park KS, Lee HK and Cho YM: Gene expression pattern in transmitochondrial cytoplasmic hybrid cells harboring type 2 diabetes-associated mitochondrial DNA haplogroups. PloS One 6: e22116, 2011. 